\title{
Predictors of Accelerated Mortality of Tb/Hiv Co- Infected Patients on Art in Mekelle, Ethiopia: an 8 Years Retrospective Follow- Up Study
}

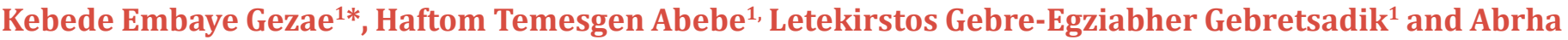 \\ Kidu Gebremeskel ${ }^{2}$
}

${ }^{1}$ Department of Biostatistics, Mekelle University: Mekelle, Ethiopia

${ }^{2}$ Department of Water Resources and Irrigation Engineering, Aksum University (Shire Campus): Shire, Ethiopia

*Corresponding author: Kebede Embaye Gezae, Department of Biostatistics, School of Public Health, College of Health Sciences, Mekelle University: Mekelle, Ethiopia.
Received Date: December 10, 2019

Published Date: December 19, 2019

\section{Abstract}

Background: Ethiopia is a country with high burden of tuberculosis and human immunodeficiency virus (TB-HIV) dual infections in the world. However, little was known so far on predictors of time to death among TB/HIV co-infected adults in the study setting in particular. Therefore, this study was aimed at filling this gap in the region particularly.

Methods: A hospital based retrospective cohort study design was employed on 305 Tuberculosis and Human Immunodeficiency Virus (TB/ HIV) co-infected adults who have started Anti-Retroviral Therapy (ART) from January 2009 to December 2016 at two governmental hospitals in Mekelle, Ethiopia. Multivariable cox regression analysis was applied to identify statistically significant predictors of time to death (P $\leq 0.05)$. Finally, Adjusted Hazard Ratio (AHR) and 95\% Confidence Interval (CI) were interpreted and reported in the final cox model.

Results: Overall, 70 of 305 (23.0\%) TB/HIV co-infected adults were died during the entire follow-up period. The study subjects (257 Active TB, 48 Latent TB) were followed for an overall median follow up time of 37 months (Interquartile Range: 10-63 months). Baseline Body Mass Index $(<18.5 \mathrm{~kg} / \mathrm{m} 2)(\mathrm{AHR}=2.427 ; 95 \% \mathrm{CI}: 1.214$ - 4.853) and Being Extra-pulmonary TB (Mixed TB) patient (AHR=2.400; 95\% CI: 0.220-0.697) were predictors of time to death. On the other hand, increasing CD4 cell count (AHR=0.995; 95\% CI: 0.991-0.999), developing drug side effects (AHR=0.369; 95\% CI: 0.194-0.701), being co-infected with Latent TB infection (AHR=0.102; 95\% CI: 1.023-0.449), completing TB treatment (AHR=0.114; 95\% CI: 0.060-0.16), and being on Cotri-moxazole Prophylactic Therapy (AHR=0.391; 95\% CI: 1.348, 4.273) had prolonged the time to death.

Conclusions: Almost one-fourth of TB/HIV co-infected patients were died with a relatively high mortality rate among those co-infected with active TB since ART initiation. Moreover, being co-infected with active TB/HIV, having low baseline BMI $(\leq 18.5 \mathrm{~g} / \mathrm{dl})$, Low CD4 cell count, not developing drug side effects, being on TB treatment, and being off CPT were shortening the time to death. Therefore, efforts have to be made to reduce malnutrition and active-TB/HIV co-infection associated accelerated mortality.

Keywords: Adults; Time to death; TB/HIV

Abbreviations: AHR: Adjusted Hazard Ratio; ART: Anti-Retroviral Therapy; BMI: Body Mass Index; CPT: Co-trimoxazole Preventive Therapy; CHR: Crude Hazard Ratio; CI: Confidence Interval; EPTB: Extra-Pulmonary Tuberculosis; HIV: Human Immunodeficiency; Virus HR: Hazard Ratio; IPT: Isoniazid Preventive Therapy; IQR: Interquartile Range; LTBI: Latent TB Infection; OI/s: Opportunistic Infection; PLWHIV: People Living with HIV; PMs: Person Months; PTB: Pulmonary Tuberculosis; SSA: Sub-Saharan Africa; TB: Tuberculosis; WHO: World Health Organization

\section{Introduction}

Tuberculosis (TB) is the most common opportunistic infection among People Living with Human Immunodeficiency Virus (PLWHIV), and persons co-infected with HIV and TB are highly vulnerable to death as a result of the bidirectional relationship and synergism of the two infections [1]. The risk of death in co-infected individuals is also approximately twice that of HIV infected individuals alone even considering CD4 cell count and AntiRetroviral therapy (ART) into account [2]. HIV is the first and TB is the second leading cause of death from infectious disease statistics world widely. Globally, above one-third of the world population 
were estimated to be latently infected with TB [3]. In the year 2014, above 9 million people developed TB, of which 12\% were co-infected with HIV [4]. In 2015, $33 \%$ of HIV deaths were due to TB evidencing that it is still a leading cause of mortality among PLWHIV [5].

Similarly, retrospective cohort studies employed in different parts of Ethiopia have been reported. The positive relationship between HIV and TB. For instance, a retrospective cohort study in Ethiopia has reported that $9.0 \%$ of HIV patients on ART were estimated to develop TB [6]. Another study from Amhara region, Ethiopia has also revealed that above one-fourth of HIV patients on ART were co-infected with TB [7]. Another study took place in Bahir Dar reported almost one -third of TB patients were also experienced to have HIV and above one-fifth of the TB-HIV cases were fatal [8]. Furthermore, accelerated TB/HIV deaths were significantly associated with low baseline CD4 cell count, ambulatory or bedridden functional status, low baseline weight, advance baseline clinical stage, absence co-trimoxazole prophylaxis (CPT), and co-existence of other OIs in most of the studies [6-12].

According to the national annual performance report of Ethiopia, the ART coverage of TB/HIV co-infected patients was as low as $70 \%$ [13]. However, the predictors of accelerated mortality of TB/HIV co-infected patients were not yet fully investigated. Thus, this study was aimed at investigating predictors of accelerated mortality of TB/HIV co-infected patents in the study setting, in particular.

\section{Methods}

\section{Study aim, design and setting}

A facility based retrospective follow-up study was conducted at two governmental hospitals-Ayder Comprehensive Specialized Hospital and Mekelle Hospital - to investigate predictors of accelerated mortality of TB/HIV co-infected patients on ART from $2009-2016$.

\section{Sample size determination and enrollment procedure}

The minimum sample sized was estimated using a formula for survival data analysis based on the following important assumptions - 95\% Confidence level, $80 \%$ optimum statistical power, CPT as major exposure variable from a similar study conducted in Northwest Ethiopia [8] (on CPT as exposed groups denoted by q1 (0.88) and Off CPT as non-exposed groups denoted by q0 (0.12)), HR (CPT) $=0.33$, and cumulative incidence of death (p) $=0.22$. The minimum sample size was estimated to be 278 as follows. We, eventually, included 305 TB/HIV co-infected patients all consecutively. The overall enrollment procedure of the study subjects was summarized in (Figure 1).

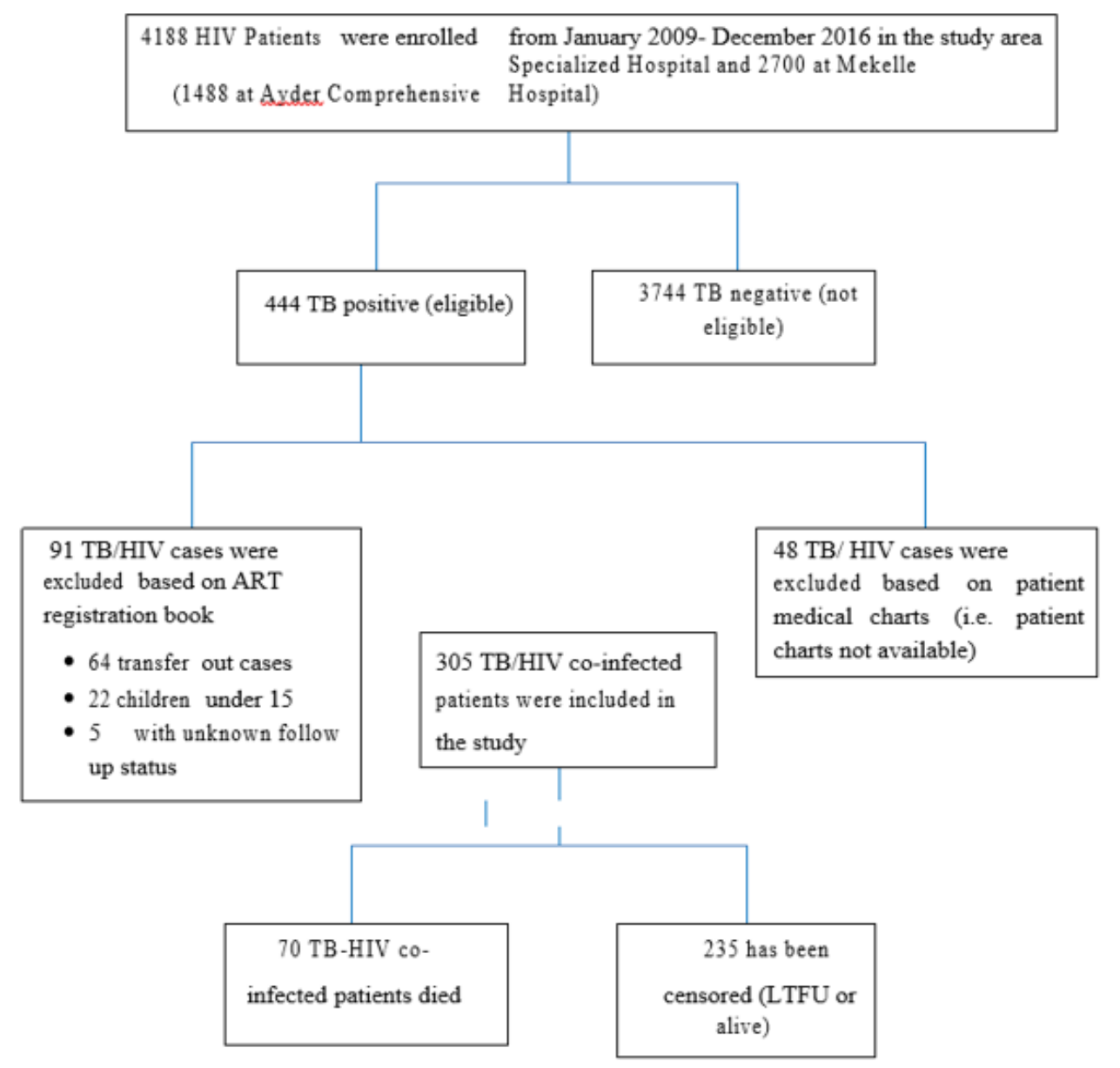

Figure 1: Overall enrollment of TB/HIV co-infected patients on ART at two governmental hospitals in Mekelle, Ethiopia $(n=305)$. 


\section{Data collection procedure and study variables}

Data were extracted from ART registration book and patient medical charts. The data collection tool consists of the following important information: 1) background characteristics of patients and 4) ART follow up status (i.e. dead, alive, stop, drop or lost to follow-up) where death was our event of interest and time to death was our outcome variable.

\section{Statistical data analysis}

First, Data were entered, coded, cleaned and then analyzed in STATA (version 12) statistical tool. Data were declared to survival time data prior to statistical data analysis. In descriptive statistics, median (IQR), and frequency (\%) were computed for numeric and categorical variables respectively. A multiple cox regression model was fitted to identify statistically significant predictors of accelerated mortality due to TB/HIV co-infection and related factors based on the estimated Adjusted Hazard Ratio (AHR) and its 95\% Confidence Interval (CI).

\section{Results}

General description of TB/HIV co-infected patients by their outcome status A total of 305 TB/HIV co-infected patients were

Table 1: General characteristics by mortality status of TB/HIV co-infected patients on ART at two governmental hospitals in Mekelle, Ethiopia ( $n=305$ ).

\begin{tabular}{|c|c|c|c|}
\hline Variable & Deaths (\%) & Mortality rate $[95 \% \mathrm{CI}]$ per $1000 \mathrm{PMs}$ & P-Value \\
\hline \multicolumn{3}{|c|}{ Sex } & \multirow{3}{*}{0.5376} \\
\hline Male & $32(21.6)$ & $6.2[4.5,8.6]$ & \\
\hline Female & $38(24.2)$ & $5.5[3.9,7.7]$ & \\
\hline \multicolumn{3}{|c|}{ Baseline Age (Years) } & \multirow{3}{*}{0.9425} \\
\hline$>35.0$ & $30(23.3)$ & $5.8[4.1,8.3]$ & \\
\hline$\leq 35.0$ & $40(22.7)$ & $5.9[4.3,8.0]$ & \\
\hline \multicolumn{3}{|c|}{ Marital Status } & \multirow{4}{*}{0.8698} \\
\hline Single & $17(24.6)$ & $6.8[4.2,10.9]$ & \\
\hline Married & $29(22.5)$ & $5.5[3.8,7.9]$ & \\
\hline *Others & $20(22.4)$ & $5.8[3.9,8.6]$ & \\
\hline \multicolumn{3}{|c|}{ Educational Level } & \multirow{5}{*}{0.5145} \\
\hline No formal education & $13(20.0)$ & $4.7[2.8,8.2]$ & \\
\hline Primary & $26(28.9)$ & $7.4[5.1,10.9]$ & \\
\hline Secondary & $24(20.2)$ & $5.4[3.6,8.1]$ & \\
\hline T ertiary & $7(22.6)$ & $5.5[2.6,11.5]$ & \\
\hline \multicolumn{3}{|c|}{ Place of Residence } & \multirow{3}{*}{0.8716} \\
\hline Urban & $50(22.5$ & $5.8[4.4,7.6]$ & \\
\hline Rural & $20(24.1)$ & $6.1[3.9,9.4]$ & \\
\hline \multicolumn{3}{|c|}{ ART Follow up site } & \multirow{3}{*}{0.9425} \\
\hline ACSH & $31(23.3)$ & $6.2[4.3,8.8]$ & \\
\hline Mekelle Hospital & $39(22.7)$ & $5.6[4.1,7.7]$ & \\
\hline \multicolumn{3}{|c|}{ Baseline BMI $\left(\mathrm{kg} / \mathrm{m}^{2}\right)$} & \multirow{3}{*}{$<0.0001$} \\
\hline$>=18.5$ & $11(10.2)$ & $2.2[1.2,3.9]$ & \\
\hline$<18.5$ & $59(30.0)$ & $8.6[6.7,11.1]$ & \\
\hline \multicolumn{3}{|c|}{ Functional Status } & \multirow{4}{*}{0.0003} \\
\hline Working & $22(15.6)$ & $3.5[2.3,5.3]$ & \\
\hline Ambulatory & $28(25.5)$ & $6.7[4.6,9.7]$ & \\
\hline Bedridden & $20(37.0)$ & $13.7[8.8,21.2]$ & \\
\hline
\end{tabular}

included in the current study. The median age of the participants at enrollment was 35 (IQR: 29 - 40 Years). Overall, 70 of 305 TB/HIV co-infected patients on ART were died during the entire follow-up period with a median time to death of 9 months and the total PMs contributed by the entire cohort was 11, 933. Based on their outcome status, 32 (21.6\%), 29 (22.5) and 26 (28.9) male, married and with no formal education TB/HIV co-infected patients - developed the event of interest in the current study.

The median baseline BMI and CD4 cell count of the participants were 7.4 (IQR: 15.6 - $19.3 \mathrm{~kg} / \mathrm{m} 2$ ) and 86 (IQR: 38-167 cells/ $\mathrm{mm} 3)$, respectively. Based on the mortality rate, the mortality rate of TB/HIV co-infected patients with low BMI (18.5 kg/m2) was roughly 4 times as compared to normal or high BMI (>=18.5 kg/ $\mathrm{m} 2$ ). The mortality rates were reported to be $13.7,8.3$ and 10.4 new TB/HIV co-infection deaths per 1000 PMs in patients with bedridden functional status, clinical stage IV and Anemic status (baseline Hemoglobin $<=11.0 \mathrm{~g} / \mathrm{dl}$ ), respectively. Furthermore, the mortality rates were reported to be 10.4 and 13.5 deaths per 1000 PMs among TB/HIV co-infected patients who were - anemi cat baseline and off CPT, respectively (Table 1 ). 


\begin{tabular}{|c|c|c|c|}
\hline \multicolumn{3}{|c|}{ WHO Clinical Stage } & \multirow{4}{*}{0.0138} \\
\hline I or II & $3(9.4)$ & $2[0.7,6.3]$ & \\
\hline III & $26(19.9)$ & $4.7[3.2,7.0]$ & \\
\hline IV & $41(28.9)$ & $8.3[6.1,11.2]$ & \\
\hline \multicolumn{3}{|c|}{ Baseline Hemoglobin (g/dl) } & \multirow{3}{*}{0.0001} \\
\hline$>11.0$ & $32(16.8)$ & 3. $9[2.7,5.5]$ & \\
\hline$\leq 11.0$ & $38(33.3)$ & $10.4[7.5,14.3]$ & \\
\hline \multicolumn{3}{|c|}{ ART Regimen } & \multirow{4}{*}{0.7566} \\
\hline 2nd line & $6(31.6)$ & $5.8[2.6,13.0]$ & \\
\hline 1st line (AZT based) & $18(31.0)$ & $5.9[3.7,9.3]$ & \\
\hline 1st line (T DF based & $46(20.2)$ & $5.9[4.4,7.8]$ & \\
\hline \multicolumn{3}{|c|}{ TB diagnosis/Infection site } & \multirow{3}{*}{0.0003} \\
\hline PT B & $19(14.4)$ & $3.1[2.0,4.8]$ & \\
\hline EPT B/Mixed & $51(29.5)$ & $8.8[6.9,11.6]$ & \\
\hline \multicolumn{3}{|c|}{ History of $0 \mathrm{I} / \mathrm{s}$ reported } & \multirow{3}{*}{0.0464} \\
\hline Yes & $62(12.9)$ & $3.2[1.6,6.4]$ & \\
\hline No & $8(25.5)$ & $6.6[5.1,8.4]$ & \\
\hline \multicolumn{3}{|c|}{ TB Treatment Completion before death } & \multirow{3}{*}{$<0.0001$} \\
\hline Yes & $44(17.4)$ & $3.8[2.8,5.1]$ & \\
\hline No & $26(50.0)$ & $59.5[40.5,87.4]$ & \\
\hline \multicolumn{3}{|c|}{ TB Type at Enrollment } & \multirow{3}{*}{0.003} \\
\hline LT BI & $2(4.2)$ & $0.8(0.2,3.1]$ & \\
\hline Active T B Disease & $68(26.5)$ & $7.2[5.7,9.2]$ & \\
\hline \multicolumn{3}{|c|}{ CPT Status } & \multirow{3}{*}{0.0034} \\
\hline On & $51(20.1)$ & $4.6[3.5,6.1]$ & \\
\hline Off & $19(37.3)$ & $13.5[9.0,20.4]$ & \\
\hline
\end{tabular}

*others $=$ Widowed/Divorced/Separated

Predictors of accelerated mortality of TB-HIV co-infected patients

Table 2: Predictors of accelerated mortality of TB/HIV co-infected patients on ART at two governmental hospitals in Mekelle, Ethiopia ( $n=305$ ).

\begin{tabular}{|c|c|c|c|}
\hline Variable & CHR [95\% CI] & AHR $[95 \% \mathrm{CI}]$ & P-Value \\
\hline \multicolumn{4}{|c|}{ Baseline BMI $\left(\mathrm{kg} / \mathrm{m}^{2}\right)$} \\
\hline$\geq 18.5$ & 1 & 1 & \\
\hline$<18.5$ & $3.617[2.476,5.284]$ & $3.529[1.797$, & $<0.001^{* *}$ \\
\hline Baseline CD4 count (Cells/mm³) & $0.993[0.991,0.995]$ & $0.994[0.990,0.998]$ & $0.004^{* *}$ \\
\hline \multicolumn{4}{|c|}{ TB treatment completion } \\
\hline No & 1 & 1 & \\
\hline Yes & $0.093[0.068,0.127]$ & $0.103[0.055,0.193]$ & $<0.001^{* *}$ \\
\hline \multicolumn{4}{|c|}{ TB diagnosis/Infection site } \\
\hline PTB & 1 & 1 & \\
\hline EPTB/M ixed & $2.575[1.887,3.515]$ & $1.753[1.010,3.041]$ & $0.046^{*}$ \\
\hline \multicolumn{4}{|c|}{ TB type } \\
\hline Active TB Disease & 1 & 1 & \\
\hline LTBI & $0.119[.0519,0.271]$ & $0.111[0.027,0.462]$ & $0.002^{* *}$ \\
\hline \multicolumn{4}{|c|}{ CPT Status } \\
\hline Off & 1 & 1 & \\
\hline On & $0.347[0.258,0.465]$ & $0.542[0.305,0.964]$ & $0.037^{*}$ \\
\hline
\end{tabular}

$1.000=$ Reference group*: significant at $5 \%,{ }^{* *}$ : significant at $0.1 \%$ level of significance

\section{Discussion}

Overall, 70 of 305 (23.0\%) of TB/HIV co-infected patients were died with an incidence rate of 5.9 per 1000 PMs. The mortality rate in the current study is lower than similar study conducted in Bahirdar [8] which reported - 4.1 new cases per 100 PMs. The magnitude of TB-HIV and related mortality was higher as compared to similar 
studies in Bahirdar, Hawassa, Jimma, Ambo, Bahirdar, and Ethiopia which had been reported a TB-HIV mortality rate of 18\%, 13.5\%, $20.2 \%, 15.8 \%, 18 \%$, and $15.5 \%$, respectively $[6,8-12,14,16,17]$.

Based on the baseline BMI, 59 of 197 (29.9\%) of TB/HIV coinfected patients with Baseline BMI $(<18.5 \mathrm{~kg} / \mathrm{m} 2)$ were died and hence, it had increased the risk of mortality by approximately 3.5 folds. TB/HIV co-infected patients with low BMI $(<18.5 \mathrm{~kg} / \mathrm{m} 2)$ had approximately four times more likely to die than their comparative group (BMI $\geq 18.5 \mathrm{~kg} / \mathrm{m} 2$ ). One study from Lesotho supported the inverse relationship between BMI and mortality [18].

Similarly, baseline CD4 cell count was statistically negatively associated with accelerated mortality of TB/HIV co-infected patients on ART, which is consistent with otheri $\mathrm{n} \mathrm{d} \mathrm{e} \mathrm{p} \mathrm{e} \mathrm{n} \mathrm{d} \mathrm{e} \mathrm{n} \mathrm{t}$ similar studies report from Ethiopia (Ambo and Bahirdar) that concluded the protective effect of increased CD4 cell count against the accelerated mortality $[8,10,19]$.

TB diagnosis was statistically associated with mortality that EPTB /mixed TB increased the risk of mortality by 1.75 folds. The finding of our study was consistent with a study conducted in Bahirdar, Ethiopia [8]. Unlikely, the results of our study was different from the findings reported from Ambo Referral Hospital [10]. EPTB or mixed TB cases might have greater chance to be estimated to different body organs which may result in treatment failure and poor treatment outcomes of Multi-Drug Resistant TB (MDR-TB).

Half (50.0\%) of TB/HIV co-infected patients were died during TB Treatment phase or before TB treatment completion that was supported by another study conducted in Ethiopia [6]. This might be probably due to the fact that regular completion of TB treatment would be broken the synergistic positive relationship between TB and HIV dual infections. Finally, the most important predictor variable in the final cox model was CPT. TB/HIV co-infected patients on CPT had better survival and reduced the risk of mortality by approximately 56\%. Our finding was in line with similar studies conducted in Zambia, Lusaka, Uganda, and Bahirdar [8,16,20,21]. In addition, a systematic review and meta-analysis study had reported the $50 \%$ protective effect of CPT among patients co-infected with TB and HIV [22].

\section{Conclusion}

Almost 1 in 4 of TB/HIV co-infected patients was died. Moreover, having low baseline BMI $\quad(<18.5 \mathrm{~g} / \mathrm{dl})$, Low CD4 cell count, failure to complete TB treatment/being on treatment, and being off CPT were accelerating the mortality. Therefore, efforts have to be made to reduce malnutrition associated accelerated mortality and IPT should be also taken properly by all LTB co-infected HIV to minimize active-TB/HIV associated accelerated mortality.

\section{Acknowledgement}

None.

\section{Conflict of Interest}

No conflict of interest. 